\title{
Éducation thérapeutique du patient âgé : la pièce manquante ?
}

\author{
Therapeutic Education and the Elderly Patient: Are we Missing a Trick?
}

\section{H. Villars}

(C) Lavoisier SAS 2014

S'il ne fait plus de doute que l'éducation thérapeutique du patient — qui s'entend comme « un ensemble de pratiques visant à permettre au patient l'acquisition de compétences pour prendre en charge sa maladie activement, en partenariat avec les soignants $"$ - a toute sa place dans le soin au patient atteint de maladie chronique, la question de sa mise en œuvre chez le sujet âgé reste complexe. En effet, elle peut théoriquement s'adresser à la fois au malade atteint d'une affection chronique qui avance en âge, au patient qui voit apparaître avec l'âge une maladie chronique, mais aussi au sujet âgé en risque de perte d'autonomie, comme cela est d'ailleurs actuellement recommandé par la Haute Autorité de santé. Autant de problématiques diverses et d'approches, de fait, différentes.

Les croyances répandues selon lesquelles les comportements en général, et de santé en particulier, ne peuvent plus être changés et qu'il est impossible de faire naître la motivation au changement chez le patient ou son proche sont les freins majeurs à la mise en œuvre d'une démarche éducative dans cette population particulièrement hétérogène. Par ail- leurs, pour certains, le vieillissement en lui-même entraîne une perte d'autonomie qui revêt un caractère « inéluctable », contre lequel aucune intervention ne peut lutter.

Pourquoi éduquer le patient âgé ? D'une part parce que le patient âgé, comme tout autre, est en demande d'être entendu dans ses besoins et ses aspirations, et d'être actif dans les soins qui lui sont prodigués. D'autre part, parce que tout processus d'éducation thérapeutique vise à rendre le patient plus autonome, chose particulièrement recherchée ici. Enfin, parce que le vieillissement est un phénomène éminemment dynamique, fait de « deuils » successifs, périodes paradoxalement et finalement propices au changement.

En conclusion, à l'heure où l'Académie nationale de médecine qualifie l'éducation thérapeutique du patient de pièce maîtresse pour faire évoluer le système de santé, nous avons jugé opportun de vous faire partager, dans ce numéro spécial, les différents points de vue et expériences d'équipes de cliniciens et de chercheurs expérimentés dans le domaine, appartenant pour la plupart au groupe de réflexion de la Société française de gériatrie et gérontologie sur le sujet.

H. Villars $(\bowtie)$

Gérontopôle, CHU de Toulouse Purpan, hôpital de jour

gériatrique, pavillon Junod, 170, avenue de Casselardit, F-31300

Toulouse, France

e-mail : villars.h@chu-toulouse.fr 